\title{
Transcript levels of different cytokines and chemokines correlate with clinical and endoscopic activity in ulcerative colitis
}

\author{
Alexandra Zahn*1, Thomas Giese ${ }^{2}$, Max Karner ${ }^{1}$, Annika Braun${ }^{1}$, Ulf Hinz ${ }^{3}$, \\ Wolfgang Stremmel ${ }^{1}$ and Robert Ehehalt ${ }^{1}$
}

\begin{abstract}
Address: ${ }^{1}$ Department of Gastroenterology, University Hospital Heidelberg, Im Neuenheimer Feld 410, 69120 Heidelberg, Germany, ${ }^{2}$ Institute of Immunology, University Heidelberg, Im Neuenheimer Feld 305, 69120 Heidelberg, Germany and 3Unit for Documentation and Statistics of the Department of Surgery, University of Heidelberg, Heidelberg, Germany

Email: Alexandra Zahn* - alexandra.zahn@med.uni-heidelberg.de; Thomas Giese - thomas.giese@urz.uni-heidelberg.de; Max Karner - max.karner@med.uni-heidelberg.de; Annika Braun - annika.braun@med.uni-heidelberg.de; Ulf Hinz - ulf.hinz@med.uniheidelberg.de; Wolfgang Stremmel - wolfgang.stremmel@med.uni-heidelberg.de; Robert Ehehalt - robert.ehehalt@med.uni-heidelberg.de

* Corresponding author
\end{abstract}

Published: 9 February 2009

BMC Gastroenterology 2009, 9:13 doi:10.1/86/147|-230X-9-13
Received: 16 December 2007

Accepted: 9 February 2009

This article is available from: http://www.biomedcentral.com/I47I-230X/9//3

(C) 2009 Zahn et al; licensee BioMed Central Ltd.

This is an Open Access article distributed under the terms of the Creative Commons Attribution License (http://creativecommons.org/licenses/by/2.0), which permits unrestricted use, distribution, and reproduction in any medium, provided the original work is properly cited.

\begin{abstract}
Background: A definition of disease activity in ulcerative colitis (UC) is difficult. The clinical activity index (CAI) is only an indirect assessment tool of bowel inflammation and the endoscopic activity index (EAl) sometimes cannot reflect the severity of disease to the full extent. Therefore, there is a need for an objective means to quantify inflammatory activity in mucosal biopsies. In our study, we wanted to examine the correlation between transcript levels of interleukin 8 (CXCL8), interferon $\gamma$ inducible protein 10 (CXCLI0), myeloid-related protein 14 (calgranulin B), macrophage inflammatory protein $2 \alpha(C X C L 2)$ with CAl and EAI in UC.
\end{abstract}

Methods: Cytokine and chemokine transcripts were quantified using real-time PCR in 49 mucosal biopsies from 27 different patients with UC. Cytokine transcript levels were correlated with CAI and EAI.

Results: There was a statistically significant positive correlation between CXCL8 $(r=0.30 ; p<$ $0.05)$, CXCLI0 ( $r=0.40 ; p<0.02)$, calgranulin $B(r=0.36$; $<<0.03)$, CXCL2 $(r=0.31 ; p<0.05)$ and CAl. Concerning EAl significant positive correlations for CXCL8 $(r=0.37 ; p<0.02), C X C L I 0$ $(r=0.33$; $p<0.04)$, calgranulin $B(r=0.31 ; p<0.05)$ and CXCL2 $(r=0.44 ; p<0.05)$ were found. Low clinical and endoscopic activity was accompanied by low cytokine levels whereas high $\mathrm{CAl}$ and EAI were associated with high cytokine levels.

Conclusion: From our data, we conclude that real-time PCR quantification of CXCL8, CXCLI0, calgranulin $\mathrm{B}$ and $\mathrm{CXCL2}$ in colonic biopsies is a simple and objective method for grading inflammation of intestinal mucosa in UC. CXCL8, CXCLI0, calgranulin B and CXCL2 might be used as biomarkers and thus as an objective tool especially in clinical trials to evaluate antiinflammatory and immunomodulatory regimens.

\section{Background}

Inflammatory bowel disease (IBD) like ulcerative colitis (UC) and Crohn's disease (CD) are characterized by a relapsing and remitting clinical course. Disease activity and severity are variable and include both segmental processes with slight impairment of state of health and pancol- 
itis with extensive gastrointestinal and systemic symptoms. Thus, the definition of disease activity in UC is often difficult. The clinical activity index (CAI) is only an indirect assessment tool of bowel inflammation and the endoscopic activity index (EAI) is sometimes unable to reflect the severity of disease to the full extent.

Cytokine and chemokine mRNA expression profiles in UC have been characterized in former studies [1-3] and interleukin 8 (CXCL8), interferon $\gamma$ inducible protein 10 (CXCL10), myeloid-related protein 14 (calgranulin B) and macrophage inflammatory protein $2 \alpha$ (CXCL2) were identified as important inflammatory mediators. However, transcript levels of these cytokines and chemokines in the blood can not reflect the inflammatory activity in the gut accurately $[2,3]$. But a correlation between mucosal mRNA levels of specific cytokines and chemokines measured by real-time PCR and mucosal inflammation has been shown for CD patients and in patients with ileopouch anal anastomosis (IPAA) [4,5]. However, so far no data concerning the correlation between transcript levels of the above mentioned selected pro-inflammatory cytokines CXCL8, CXCL10, calgranulin B and CXCL2 in mucosal biopsies and disease activity indices of UC patients exist. Previous studies either evaluated changes in other than the above mentioned cytokines/chemokines like Raddatz et al. [6] who showed a positive correlation between interleukin 6 (IL-6) mRNA transcript levels and CAI in patients with pancolitis or used methods like Northern Blot analysis [7], in situ hybridization [8], ELISA [7,9-12] or immunohistochemistry [8] which are not considered reliable for quantification of mucosal cytokine gene expression. As an adequate treatment of UC requires an objective assessment of mucosal inflammation, there is a need for a simple and objective means to quantify inflammatory activity. The recent development of real time PCR methodology should allow a routine and reliable quantification of PCR products. Therefore, we measured the transcript levels of CXCL8, CXCL10, calgranulin $\mathrm{B}$ and CXCL2 in biopsies of UC patients in this study and correlated them to CAI and EAI.

Thus, the aim of this study was to establish several biomarkers which can reflect mucosal inflammation in UC.

\section{Methods \\ Patients}

All biopsies were taken from patients who participated in one of our clinical studies $[13,14]$. The biopsies were taken from the colon section $30-40 \mathrm{~cm}$ ab ano to get a standardization for our study. Our studies were approved by the institutional Ethics Committee of the University of Heidelberg. CAI and EAI were assessed according to Rachmilewitz [15] (tables 1 and 2). Altogether, 49 biopsies
Table I: Clinical Activity Index (CAI) according to Rachmilewitz.

\begin{tabular}{|c|c|}
\hline \multicolumn{2}{|l|}{ Number of stools weekly } \\
\hline$<18$ & 0 \\
\hline $18-35$ & I \\
\hline $36-60$ & 2 \\
\hline$>60$ & 3 \\
\hline \multicolumn{2}{|l|}{ Blood in stools (based on weekly average) } \\
\hline None & 0 \\
\hline Little & I \\
\hline A lot & 2 \\
\hline \multicolumn{2}{|c|}{ Investigator's global assessment of symptomatic state } \\
\hline Good & 0 \\
\hline Average & I \\
\hline Poor & 2 \\
\hline Very poor & 3 \\
\hline \multicolumn{2}{|l|}{ Abdominal pain/cramps } \\
\hline None & 0 \\
\hline Mild & I \\
\hline Moderate & 2 \\
\hline Severe & 3 \\
\hline \multicolumn{2}{|l|}{ Temperature due to colitis } \\
\hline$<38^{\circ} \mathrm{C}$ & 0 \\
\hline$>38^{\circ} \mathrm{C}$ & 1 \\
\hline \multicolumn{2}{|l|}{ Extraintestinal manifestations } \\
\hline Iritis & 3 \\
\hline Erythema nodosum & 3 \\
\hline Arthritis & 3 \\
\hline \multicolumn{2}{|l|}{ Laboratory Findings } \\
\hline Sedimentation rate $>50 \mathrm{~mm}$ in $\mathrm{Ist}$ hour & 1 \\
\hline Sedimentation rate $>100 \mathrm{~mm}$ in $1 \mathrm{st}$ hour & 2 \\
\hline Haemoglobin $<100 \mathrm{~g} / \mathrm{L}$ & 4 \\
\hline
\end{tabular}

from 27 different patients were examined. In table 3 patients' sociodemographic and clinical data are given. Nineteen patients were male, eight female. Patients' mean age was 37 years (range, 18-53 years). Patients' disease duration averaged out at 9 years (range, 1-23 years). Seventeen of our patients had a pancolitis, nine a left sided colitis and one patient had only proctitis. In table 4 the CAI (mean CAI, 6; range, 0-14) and the EAI (mean EAI, 7; range 2-10) assigned to each biopsy at the time of sampling are shown. Three patients had extraintestinal manifestations of UC, namely arthritis. 24 patients received 
Table 2: Endoscopic Activity Index (EAI) according to Rachmilewitz.

\begin{tabular}{ll}
\hline Granulation scattering reflected light \\
\hline No & 0 \\
Yes & 2
\end{tabular}

\begin{tabular}{ll} 
Vascular pattern & \\
\hline Normal & 0 \\
Faded/disturbed & 1 \\
Completely absent & 2
\end{tabular}

Vulnerability of mucosa

$\begin{array}{ll}\text { None } & 0 \\ \text { Slightly increased (contact bleeding) } & 2 \\ \text { Greatly increased (spontaneous bleeding) } & 4\end{array}$

Mucosal damage (mucus, fibrin, exudate, erosions, ulcer)

\begin{tabular}{ll}
\hline None & 0 \\
Slight & 2 \\
Pronounced & 4
\end{tabular}

drug treatment. A single treatment regimen with prednisolone was given to 7 patients, a single treatment regimen with 5-aminosalicylic acid (5-ASA) to 5 patients. 6 patients received prednisolone and 5-ASA, 3 patients received prednisolone and azathioprine and 3 patients were treated with prednisolone, 5-ASA and azathioprine. If one patient was included in our study twice, biopsies were taken at two different time points at intervals of at least 3 months.

\section{Real-Time RT-PCR}

Biopsies were collected in RNAlater (Ambion, Austin, TX, USA), and stored at $-20^{\circ} \mathrm{C}$ until analysis. Tissue was disrupted by one run with the RiboLyser (ThermoHYBAID, Heidelberg) in lysing matrix " $\mathrm{D}^{\prime}$ tubes (Q-BIOgen, Heidelberg) containing $400 \mu \mathrm{l}$ lysis buffer from the MagnaPure mRNA Isolation Kit II (ROCHE Diagnostics, Mannheim). The RiboLyser tubes were centrifuged at $4{ }^{\circ} \mathrm{C}$ for $1 \mathrm{~min}$ at $13000 \mathrm{rpm} .300 \mu \mathrm{l}$ of the lysate was collected and mixed with $600 \mu \mathrm{l}$ capture buffer containing oligo-dT. After centrifugation at $13000 \mathrm{rpm}$ for $5 \mathrm{~min}, 880 \mu \mathrm{l}$ of this mix was transferred into a MagnaPure sample cartridge and mRNA was isolated with the MagnaPure-LC device using the mRNA-II standard protocol. The elution volume was set to $50 \mu \mathrm{l}$.

An aliquot of $8.2 \mu \mathrm{lmRNA}$ was reversely transcribed using AMV-RT and oligo-(dT) as primer (First Strand cDNA synthesis kit, Roche) according to the manufacturers instructions in a thermocycler. After termination of the cDNA synthesis, the reaction mix was diluted to a final volume of $500 \mu \mathrm{l}$ and stored at $-20^{\circ} \mathrm{C}$ until PCR analysis.

Primer sets optimized for the LightCycler (RAS, Mannheim Germany) were developed and purchased from SEARCH-LC GmbH, Heidelberg. The PCR was performed with the LightCycler FastStart DNA Sybr GreenI kit (RAS) according to the instructions provided in the parameter specific kits. To control for specificity of the amplification products, a melting curve analysis was performed. No amplification of unspecific products was observed. The copy number was calculated from a standard curve, obtained by plotting known input concentrations of four different plasmids at log dilutions to the PCR-cycle number (CP) at which the detected fluorescence intensity reaches a fixed value. This approach dramatically reduced variations due to handling errors over several logarithmic dilution steps.

To correct for differences in the content of total RNA, the calculated copy numbers were normalized according to the average expression of two housekeeping genes, $\beta$ Actin and Cyclophilin B. Values were thus given as input adjusted copy number per $\mu \mathrm{l}$ of cDNA.

\section{Statistical analysis}

Statistical analysis was performed using SAS software (Release 9.1, SAS Institute, Cary; NC, USA). Results are described as medians, means and ranges. Correlations between cytokine/chemokine transcript levels and CAI and EAI were analyzed using the Pearson correlation coefficient (r) and the corresponding probability value (p). Multiple linear regression analysis was performed to examine whether the cytokine/chemokine transcript levels were independently associated with CAI and EAI. To control a possible bias by more than one biopsy of a person the generalized estimating equation (GEE) method was used with an independent and an exchangeable work-

Table 3: Patients' characteristics

\begin{tabular}{lc}
\hline Number of Patients & 27 \\
Sex (m/f) & $19 / 8$ \\
Age (years) & $37(18-53)$ \\
Disease Duration (years) & $9(1-23)$ \\
Disease Localisation: Pancolitis/Left sided colitis/Proctitis & $17 / 9 / 1$ \\
Extraintestinal Manifestations (arthritis) & 3
\end{tabular}

Values are given as absolute numbers and means. The values in brackets give the extreme values. 
Table 4: Biopsies' characteristics

\begin{tabular}{ll}
\hline Number of Biopsies & 49 \\
Mean CAl & $6(0-14)$ \\
Mean EAI & $7(2-10)$ \\
\hline
\end{tabular}

Values are given as absolute numbers and means. The values in brackets give the extreme values.

ing correlation matrix. Two sided probability values were always computed and an effect was considered statistically significant at a value of $\mathrm{p}<0.05$.

\section{Results}

Expression of cytokine/chemokine transcripts in mucosal biopsies of UC patients

In 49 biopsies from 27 UC patients the expression of CXCL8, CXCL10, calgranulin B and CXCL2 transcripts were measured. The median number of transcript copies in all biopsies was 80 (range, 0-600) for CXCL8, 75 (range, 3-339) for CXCL10, 799 (range, 16-10517) for calgranulin B and 353 (range, 10-2484) for CXCL2.

\section{Correlation of cytokine/chemokine transcript levels with CAI and EAI}

To determine the convergence of cytokine/chemokine transcript levels with the activity of UC, the correlations between cytokine/chemokine transcript levels and clinical and endoscopic disease activity were calculated. The correlations between cytokine/chemokine transcript levels and CAI for all biopsies are shown in figures 1, 2, 3, 4. There was a statistically significant positive correlation between CXCL8 ( $\mathrm{r}=0.30 ; \mathrm{p}<0.05)$, CXCL10 ( $\mathrm{r}=0.40$; $<0.02)$, calgranulin $\mathrm{B}(\mathrm{r}=0.36$; $\mathrm{p}<0.03)$ and CXCL2 $(\mathrm{r}=$ $0.31 ; \mathrm{p}<0.05)$ with CAI. The correlations between

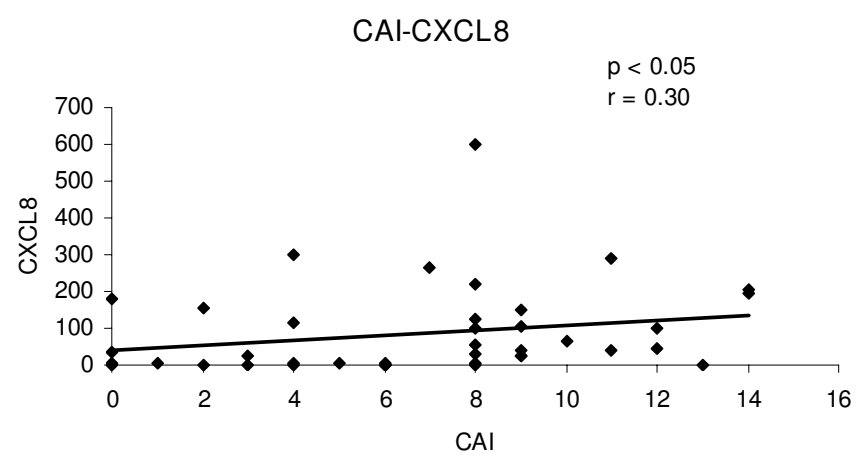

Figure I

Linear regression analysis of CXCL8 transcript levels and CAl in all biopsies. On the $x$ axis, the values of the $\mathrm{CAl}$ are given. On the $\mathrm{y}$ axis, the cytokine transcript levels are shown. Each single biopsy is characterized by I point within the graph. For the graph the related Pearson correlation coefficient $(r)$ and the corresponding probability value (p) are given.

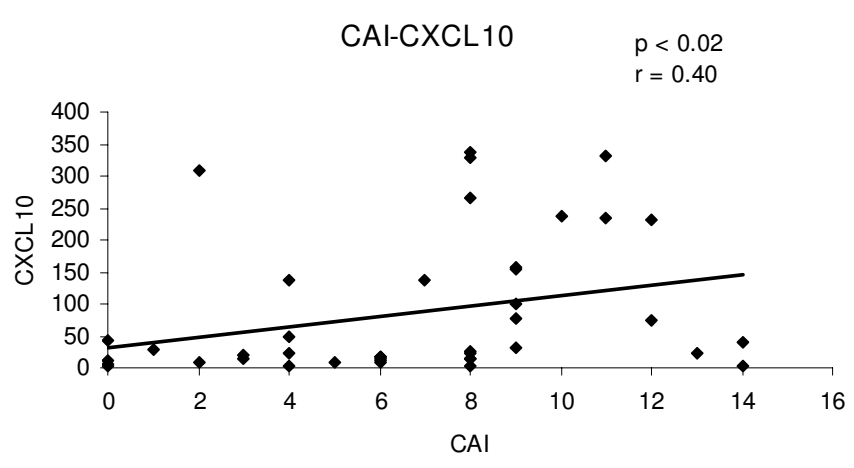

Figure 2

Linear regression analysis of CXCLI 0 transcript levels and CAI in all biopsies. On the $x$ axis, the values of the $\mathrm{CAl}$ are given. On the $\mathrm{y}$ axis, the cytokine transcript levels are shown. Each single biopsy is characterized by I point within the graph. For the graph the related Pearson correlation coefficient $(r)$ and the corresponding probability value (p) are given.

cytokine/chemokine transcript levels and EAI for all biopsies are shown in figures 5, 6, 7, 8. Concerning EAI, we could also find significant positive correlations for CXCL8 $(\mathrm{r}=0.37 ; \mathrm{p}<0.02)$, CXCL10 $(\mathrm{r}=0.33 ; \mathrm{p}<0.04)$, calgranulin B $(\mathrm{r}=0.31 ; \mathrm{p}<0.05)$ and CXCL2 $(\mathrm{r}=0.44 ; \mathrm{p}<0.05)$. Positive correlations indicate that clinical and endoscopic improvement led to a decrease in cytokine levels whereas

\section{CAl-calgranulin B}

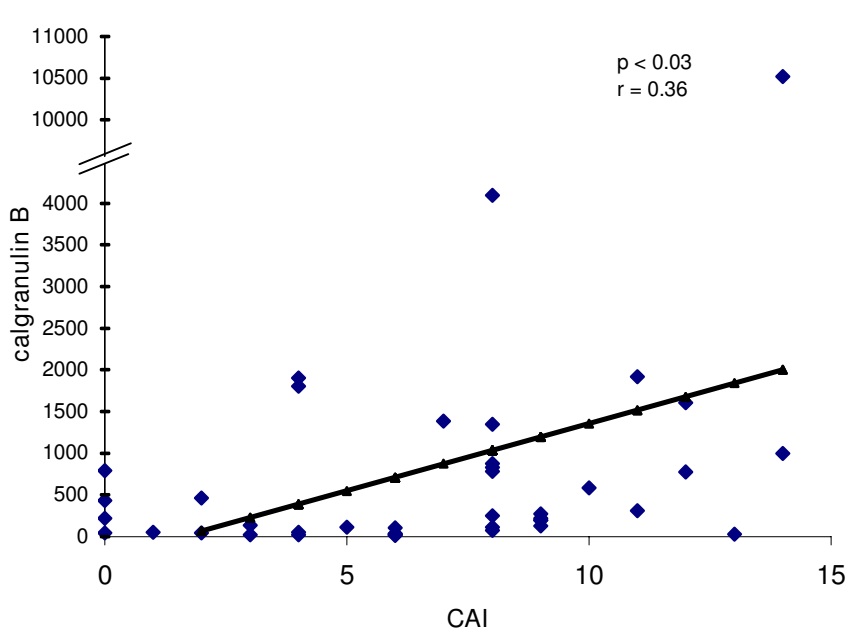

Figure 3

Linear regression analysis of calgranulin B transcript levels and CAl in all biopsies. On the $x$ axis, the values of the CAl are given. On the y axis, the cytokine transcript levels are shown. Each single biopsy is characterized by I point within the graph. For the graph the related Pearson correlation coefficient $(r)$ and the corresponding probability value (p) are given. 


\section{CAI-CXCL2}

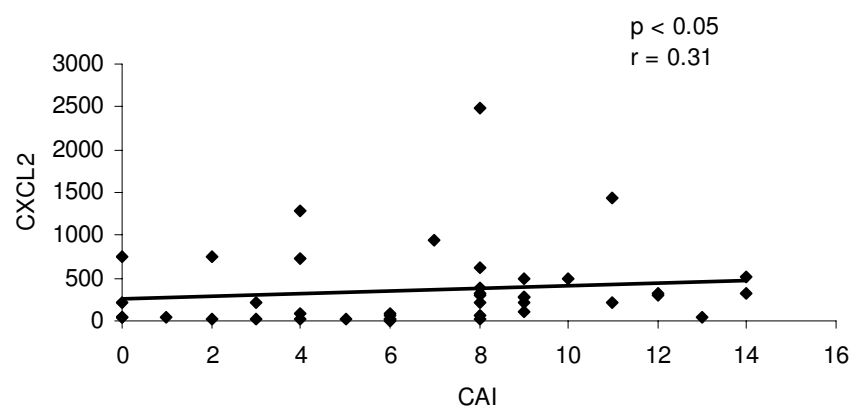

Figure 4

Linear regression analysis of CXCL2 transcript levels and CAl in all biopsies. On the $x$ axis, the values of the $\mathrm{CAl}$ are given. On the $y$ axis, the cytokine transcript levels are shown. Each single biopsy is characterized by I point within the graph. For the graph the related Pearson correlation coefficient $(r)$ and the corresponding probability value (p) are given.

a deterioration of clinical and endoscopic activity was accompanied by increased cytokine levels.

Correlation analysis was performed to examine a possible internal correlation between CXCL8, CXCL10, calgranulin $\mathrm{B}$ and CXCL2. Statistically significant positive correlations were found between CXCL8 and CXCL2 $(r=0.94 ; \mathrm{p}<$ $0.0001)$, between CXCL8 and calgranulin $B(r=0.55 ; \mathrm{p}=$ $0.0002)$ and between CXCL2 and calgranulin B $(r=0.46$; $\mathrm{p}=0.002)$. Linear regression analysis revealed then calgranulin $\mathrm{B}(\mathrm{p}=0.0008, \mathrm{p}=0.0007)$ and CXCL10 $(\mathrm{p}=$ $0.0412, \mathrm{p}=0.0873$ ) independently correlated with CAI and EAI, respectively.

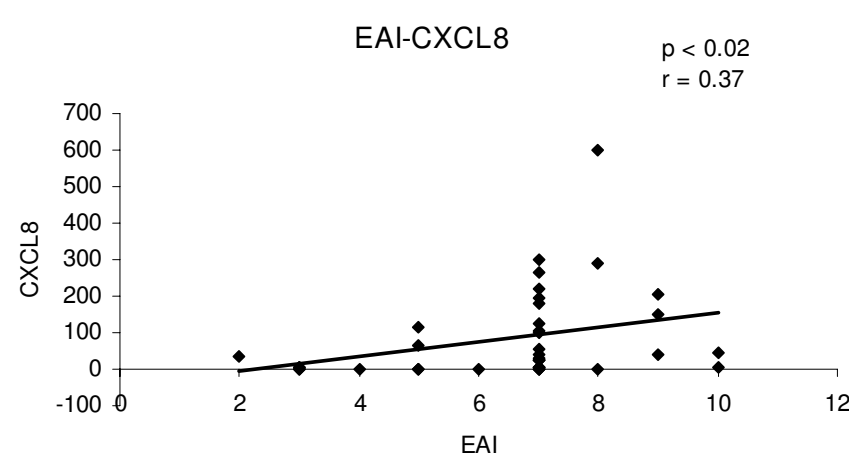

Figure 5

Linear regression analysis of CXCL8 transcript levels and EAI in all biopsies. On the $x$ axis, the values of the EAI are given. On the $y$ axis, the cytokine transcript levels are shown. Each single biopsy is characterized by I point within the graph. For the graph the related Pearson correlation coefficient $(r)$ and the corresponding probability value $(P)$ are given.

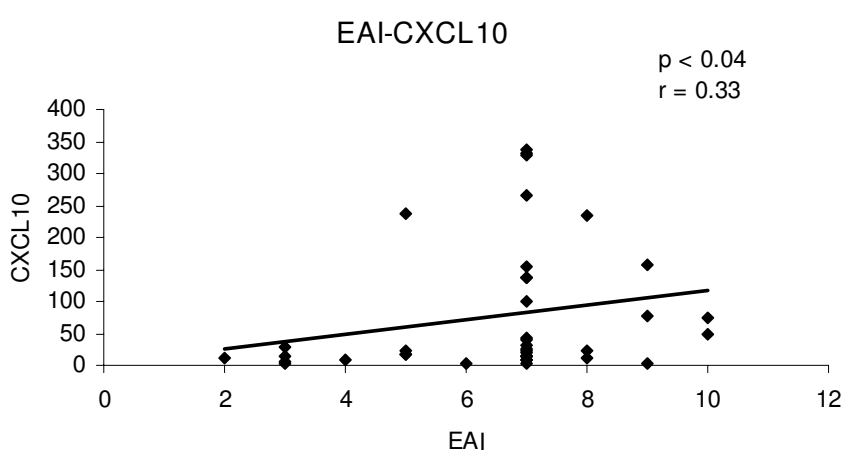

Figure 6

Linear regression analysis of CXCLI 0 transcript levels and EAl in all biopsies. On the $x$ axis, the values of the EAI are given. On the $y$ axis, the cytokine transcript levels are shown. Each single biopsy is characterized by I point within the graph. For the graph the related Pearson correlation coefficient $(r)$ and the corresponding probability value (P) are given.

Regarding the variation of CAI and EAI expressed by CXCL2, CXCL8, CXCL10, and calgranulin B the results of the linear regression for R-square were 0.360 and 0.216 , respectively.

\section{Discussion}

Although there has been progress in understanding the pathogenesis of IBD in the last decade, the etiology remains unknown. Therefore, most therapies aim at a

\section{EAl-calgranulin B}

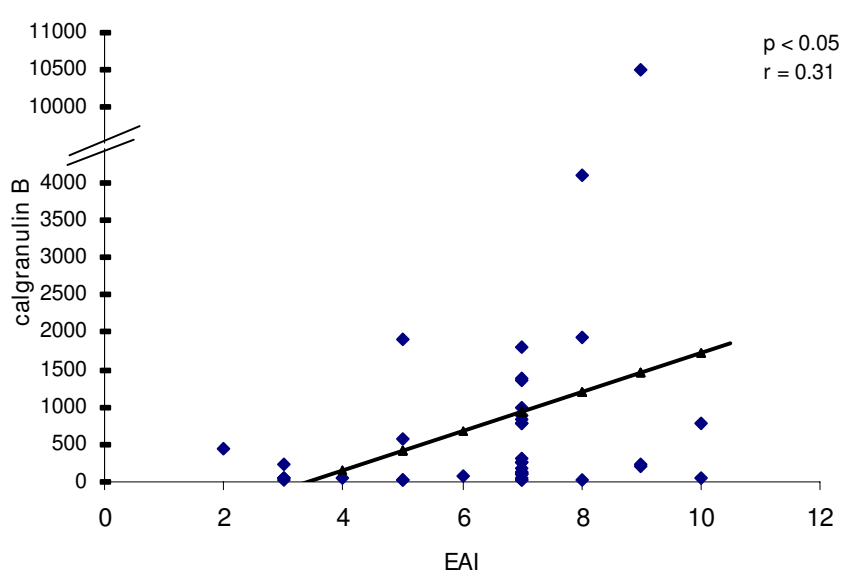

Figure 7

Linear regression analysis of calgranulin B transcript levels and EAI in all biopsies. On the $x$ axis, the values of the EAl are given. On the $y$ axis, the cytokine transcript levels are shown. Each single biopsy is characterized by I point within the graph. For the graph the related Pearson correlation coefficient $(r)$ and the corresponding probability value (P) are given. 


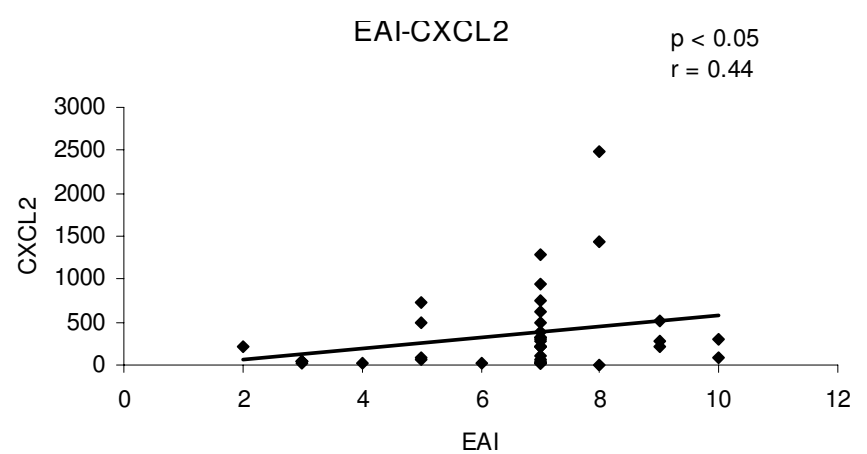

Figure 8

Linear regression analysis of CXCL2 transcript levels and $E A I$ in all biopsies. On the $x$ axis, the values of the EAI are given. On the $y$ axis, the cytokine transcript levels are shown. Each single biopsy is characterized by I point within the graph. For the graph the related Pearson correlation coefficient $(r)$ and the corresponding probability value $(p)$ are given.

symptomatic treatment of inflammation. However, objective assessment of mucosal inflammation and disease activity is often difficult. Neither CAI nor EAI, as established scores, can characterize mucosal inflammation and disease activity to the full extent. The CAI is influenced by patients' perception and may thus reflect clinical symptoms due to other factors than mucosal inflammation and the severity of endoscopic lesions often do not correlate with clinical severity.

The aim of our study was to find a simple and objective means to quantify inflammatory activity in colonic mucosa of UC patients. Therefore, we tried to establish several biomarkers, which can reflect mucosal inflammation in UC.

Thus, we measured the transcript levels of CXCL8, CXCL10, calgranulin B and CXCL2 in biopsies of UC patients and correlated them to CAI and EAI.

The new and substantial finding of our study is the significant positive correlation between transcript levels of CXCL8, CXCL10, calgranulin B and CXCL2 and both CAI and EAI.

Our results agree with the literature. For CXCL8, which is the major attractant and activator of neutrophils $[9,10,16]$, a high expression of mRNA in UC patients with high disease activity has been described [8]. Another group [17], who performed immunohistochemistry and image analysis found enhanced CXCL10 expression in UC. CXCL10 selectively attracts activated T lymphocytes. Calgranulin B is selectively secreted by human monocytes and granulocytes. Using ELISA technique an increased serum level was detected in UC patients with active disease [18]. The same group [19] could also show a greater calgranulin B production in CD in ulcerative and fissural lesions than in uninflamed areas. Concerning CXCL2, which is extremely chemotactic for neutrophils [20], an upregulated expression of mRNA and protein in inflamed gut, predominantly in UC has been shown [1].

These data from the literature strengthen our findings that CXCL8, CXCL10, calgranulin B and CXCL2 are objective indicators of disease activity and severity in UC.

\section{Conclusion}

Therefore, we conclude that measuring transcript levels of CXCL8, CXCL10, calgranulin B and CXCL2 by RT-PCR is a fast, simple and objective method to quantify mucosal inflammation in UC. This approach can give important information in addition to CAI and EAI and thus provide an objective marker of intestinal inflammation. Moreover, using this technique to monitor therapeutic effects of e. g. novel drugs can offer potential improvement in clinical research.

\section{Competing interests}

The authors declare that they have no competing interests.

\section{Authors' contributions}

$\mathrm{AZ}$ was involved in the development of methods, performed data and sample collection, data analysis and wrote the paper. TG was involved in the development of methods and carried out the quantitative RT-PCR. MK contributed to the data collection and analysis. $\mathrm{AB}$ contributed to the data collection and analysis. UH performed statistical computation. WS contributed substantially to the design of the study and to the interpretation of data. RE developed the original idea of the study, contributed substantially to the design of the study, was involved in data analysis and reviewed the manuscript finally. All authors read and approved the final manuscript.

\section{References}

I. Autschbach F, Giese T, Gassler N, Sido B, Heuschen G, Heuschen U, Zuna I, Schulz P, Weckauf H, Berger I, et al:: Cytokine/chemokine messenger-RNA expression profiles in ulcerative colitis and Crohn's disease. Virchows Arch 2002, 44I(5):500-5I3.

2. Sartor RB: Cytokines in intestinal inflammation: pathophysiological and clinical considerations. Gastroenterology 1994, 106(2):533-539.

3. Gotteland M, Lopez M, Munoz C, Saez R, Altshiller H, Llorens $P$, Brunser $O$ : Local and systemic liberation of proinflammatory cytokines in ulcerative colitis. Dig Dis Sci 1999, 44(4):830-835.

4. Stallmach A, Giese T, Schmidt C, Ludwig B, Mueller-Molaian I, Meuer SC: Cytokine/chemokine transcript profiles reflect mucosal inflammation in Crohn's disease. Int J Colorectal Dis 2004, 19(4):308-3।5.

5. Schmidt C, Giese T, Ludwig B, Menges M, Schilling M, Meuer SC, Zeuzem S, Stallmach A: Increased cytokine transcripts in pouchitis reflect the degree of inflammation but not the underlying entity. Int J Colorectal Dis 2006, 2 I (5):419-426. 
6. Raddatz D, Bockemuhl M, Ramadori G: Quantitative measurement of cytokine mRNA in inflammatory bowel disease: relation to clinical and endoscopic activity and outcome. Eur J Gastroenterol Hepatol 2005, I 7(5):547-557.

7. Kusugami K, Fukatsu A, Tanimoto M, Shinoda M, Haruta J, Kuroiwa A, Ina K, Kanayama K, Ando T, Matsuura T, et al.: Elevation of interleukin-6 in inflammatory bowel disease is macrophage- and epithelial cell-dependent. Dig Dis Sci 1995, 40(5):949-959.

8. Mazzucchelli L, Hauser C, Zgraggen K, Wagner H, Hess M, Laissue JA, Mueller C: Expression of interleukin-8 gene in inflammatory bowel disease is related to the histological grade of active inflammation. Am J Pathol 1994, 144(5):997-1007.

9. Keshavarzian A, Fusunyan RD, Jacyno M, Winship D, MacDermott RP Sanderson IR: Increased interleukin-8 (IL-8) in rectal dialysate from patients with ulcerative colitis: evidence for a biological role for IL-8 in inflammation of the colon. Am J Gastroenterol 1999, 94(3):704-7|2.

10. Mahida YR, Ceska M, Effenberger F, Kurlak L, Lindley I, Hawkey CJ: Enhanced synthesis of neutrophil-activating peptide-I/interleukin-8 in active ulcerative colitis. Clin Sci (Lond) 1992 82(3):273-275.

II. Nielsen OH, Rudiger N, Gaustadnes M, Horn T: Intestinal interleukin-8 concentration and gene expression in inflammatory bowel disease. Scand J Gastroenterol 1997, 32(10): 1028-1034.

12. Mitsuyama K, Toyonaga A, Sasaki E, Watanabe K, Tateishi H, Nishiyama T, Saiki T, Ikeda H, Tsuruta O, Tanikawa K: IL-8 as an important chemoattractant for neutrophils in ulcerative colitis and Crohn's disease. Clin Exp Immunol 1994, 96(3):432-436.

13. Stremmel W, Ehehalt R, Autschbach F, Karner M: Phosphatidylcholine for steroid-refractory chronic ulcerative colitis: a randomized trial. Ann Intern Med 2007, I 47(9):603-6I0.

14. Stremmel W, Merle U, Zahn A, Autschbach F, Hinz U, Ehehalt R Retarded release phosphatidylcholine benefits patients with chronic active ulcerative colitis. Gut 2005, 54(7):966-97I.

15. Rachmilewitz D: Coated mesalazine (5-aminosalicylic acid) versus sulphasalazine in the treatment of active ulcerative colitis: a randomised trial. Bmj 1989, 298(6666):82-86

16. Raab Y, Gerdin B, Ahlstedt S, Hallgren R: Neutrophil mucosal involvement is accompanied by enhanced local production of interleukin-8 in ulcerative colitis. Gut | 993, 34(9): | 203- 206.

17. Uguccioni M, Gionchetti P, Robbiani DF, Rizzello F, Peruzzo S, Campieri M, Baggiolini M: Increased expression of IP-I 0, IL-8, MCP-I, and MCP-3 in ulcerative colitis. Am J Pathol 1999, I55(2):331-336.

18. Lugering N, Stoll R, Schmid KW, Kucharzik T, Stein H, Burmeister G, Sorg C, Domschke W: The myeloic related protein MRP8/I4 (27E 10 antigen) - usefulness as a potential marker for disease activity in ulcerative colitis and putative biological function. Eur J Clin Invest 1995, 25(9):659-664.

19. Lugering N, Stoll R, Kucharzik T, Schmid KW, Rohlmann G, Burmeister G, Sorg C, Domschke W: Immunohistochemical distribution and serum levels of the $\mathrm{Ca}(2+)$-binding proteins MRP8, MRPI4 and their heterodimeric form MRP8/I4 in Crohn's disease. Digestion 1995, 56(5):406-4I4.

20. Widmer U, Manogue KR, Cerami A, Sherry B: Genomic cloning and promoter analysis of macrophage inflammatory protein (MIP)-2, MIP-I alpha, and MIP-I beta, members of the chemokine superfamily of proinflammatory cytokines. I Immunol 1993, I50(II):4996-50I2.

\section{Pre-publication history}

The pre-publication history for this paper can be accessed here:

http://www.biomedcentral.com/1471-230X/9/13/pre pub
Publish with Biomed Central and every scientist can read your work free of charge

"BioMed Central will be the most significant development for disseminating the results of biomedical research in our lifetime. "

Sir Paul Nurse, Cancer Research UK

Your research papers will be:

- available free of charge to the entire biomedical community

- peer reviewed and published immediately upon acceptance

- cited in PubMed and archived on PubMed Central

- yours - you keep the copyright
BioMedcentral 